\title{
Ethnologies
}

Karine Hébert et Julien Goyette (dir.). Histoire et idées du patrimoine, entre régionalisation et mondialisation. (Québec, MultiMondes, collection Cahiers de l'Institut du patrimoine de l’UQAM, 2010. Pp. 314. ISBN 978-2-89544-178-6)

\section{Louis-Simon Corriveau}

Volume 35, numéro 1, 2013

URI : https://id.erudit.org/iderudit/1026463ar

DOI : https://doi.org/10.7202/1026463ar

Aller au sommaire du numéro

Éditeur(s)

Association Canadienne d'Ethnologie et de Folklore

ISSN

1481-5974 (imprimé)

1708-0401 (numérique)

Découvrir la revue

Citer ce compte rendu

Corriveau, L.-S. (2013). Compte rendu de [Karine Hébert et Julien Goyette (dir.). Histoire et idées du patrimoine, entre régionalisation et mondialisation. (Québec, MultiMondes, collection Cahiers de l'Institut du patrimoine de l'UQAM, 2010.

Pp. 314. ISBN 978-2-89544-178-6)]. Ethnologies, 35(1), 201-204.

https://doi.org/10.7202/1026463ar d'utilisation que vous pouvez consulter en ligne. 
Karine Hébert et Julien Goyette (dir.). Histoire et idées du patrimoine, entre régionalisation et mondialisation. (Québec, MultiMondes, collection Cahiers de l'Institut du patrimoine de I’UQAM, 2010. Pp. 314. ISBN 978-2-89544-178-6)

Louvrage collectif dirigé par Karine Hébert et Julien Goyette, deux professeurs d'histoire à l'Université du Québec à Rimouski (UQAR), découle de la cinquième rencontre internationale des jeunes chercheurs en patrimoine organisée en 2009 en partenariat avec la Chaire de recherche du Canada en patrimoine urbain et l'Institut du patrimoine de l'Université du Québec à Montréal (UQAM). Cette rencontre, qui s'est effectuée comme les quatre années précédentes sous la forme d'un colloque, a amené de jeunes chercheurs de différents horizons à se regrouper autour de questionnements partagés, abordant notamment les thèmes du temps et de l'espace. L'ensemble des études présentées dans cet ouvrage riche se penche sur diverses formes d'objets patrimoniaux, passant « des plus habituels aux plus inusités », tant matériels qu'immatériels : « traditions culinaires et philosophiques, maisons, théâtres, églises, œuvres d'art, réalisations architecturales, biens archéologiques, vestiges de la période industrielle " (3). L'exploration de ces objets patrimoniaux et de ces thèmes a amené les auteurs à mettre de l'avant, directement ou non, trois formes de conciliations difficiles : « spécificité et universalisme, sauvegarde et diffusion, conservation et démocratisation ", d'abord; " les représentations, à la fois antinomiques et complémentaires, des experts, des citoyens et des touristes ", ensuite; " mémoire heureuse et mémoire honteuse, le nécessaire oubli et le devoir de mémoire ", enfin (10-11).

"Le patrimoine est une question jeune ", peut-on lire en ouverture de l'ouvrage (1). Hébert et Goyette mettent l'accent sur la proximité que le patrimoine entretient avec la jeunesse, d'une part, et le présent, d'autre part. Le patrimoine est en effet apparu comme "mode spécifique d'appréhension du temps et de la matérialité » il y a quelques siècles et n'est un champ d'études que depuis quelques décennies (2). Cette dite " jeunesse " du patrimoine comme champ d'études implique que l'objet, les concepts et les méthodes ne fassent pas encore consensus. L'introduction propose une définition globale du patrimoine qui permet au lecteur de s'aventurer dans les différentes 
études sans se sentir dérouté. La conception du patrimoine s'est élargie à travers les âges, passant d'une transmission familiale à une transmission "sociale et même mondiale » (2). Le patrimoine - et les objets patrimoniaux - apparaissent alors comme une construction historique, étant toujours situé "dans un moment et dans un lieu » (3). En tant que construction, il est également un processus, la patrimonialisation connaissant différentes étapes, ce qui nous permet de " parler d'“économie" ou d'“écosystèmes" patrimoniaux " (3), suivant les termes utilisés par Lucie K. Morisset. Le patrimoine est, de plus, façonné à la fois par différentes structures ("économiques, politiques, sociales et culturelles ») et par des acteurs individuels (7), en plus de se camper dans l'universel et dans le local, créant une distance dans la reconnaissance patrimoniale régionale et internationale (8). Si le patrimoine est souvent associé à la quête de préservation et au passé, il est également et surtout un regard obsessionnel des sociétés contemporaines pour le présent, estiment Hébert et Goyette. Les Occidentaux meublent ainsi le présent à partir de vestiges du passé, ce qui vient avec différents actes de commémoration. C'est également en ce sens que le patrimoine se rapproche de la jeunesse, puisque, de nos jours, "les héritiers s'arrogent le droit de décider du contenu de leur héritage " (2).

Cette mise en contexte conceptuelle étant faite, penchons-nous maintenant sur le contenu de cet ouvrage collectif. S'intéressant d'abord à l'arrimage entre patrimoine et politique, il aborde ensuite différents objets patrimoniaux, immatériels puis matériels, pour enfin conclure avec quelques réflexions sur le patrimoine et ses enjeux. S'il semble aisé de diviser les textes dans ces quatre parties, Hébert et Goyette n'ont toutefois pas cru bon de les présenter en suivant cette répartition, la présentation de l'ouvrage ne suivant aucun fil conducteur. On retrouve certes des liens entre les études qui sont mis de l'avant dans l'introduction, mais ces regroupements par pairs ou trios entourant des thématiques ou des angles d'approche partagés ne figurent pas tous dans les mêmes sections. Cette présentation décousue aurait été aisément justifiable si Hébert et Goyette avaient introduit les différentes études à partir d'un axe particulier, offrant une présentation fluide et synthétique. Mais l'éclectisme de l'introduction est frappant, en plus d'être assumé, pourrait-on croire. La présentation des différents regroupements de textes défile en effet à travers les " de son côté ", "d'autres thèmes ", "d'autres études ", 
"d'autres cas », etc. De plus, n'étant pas présentées par partie, les études ne le sont pas davantage en fonction de leur ordre d'apparition dans l'ouvrage collectif. On passe alors de trois textes figurant dans la troisième partie (le second, le premier puis le quatrième), au dernier de la première partie, pour ensuite sauter au premier de la quatrième partie, et ainsi de suite. Avec un tel casse-tête, il devient difficile de se faire une idée des différentes études qui constituent cet ouvrage collectif, mais surtout des liens qui les unissent.

Il demeure tout de même que, même si le texte introductif ne rend pas hommage au découpage des parties et à ce qui crée leur unité, les textes cadrent souvent bien avec ceux qui les entourent, même si certains rapprochements sont parfois plus timides. Les textes demeurent d'ailleurs, pour la plupart, épurés, sans esbroufe ni jargon, ce qui amène une belle qualité d'ensemble et évite une trop grande rupture entre les différentes études sur le plan de la forme.

Le lien entre patrimoine et politique mis de l'avant dans la première partie amène les auteurs à proposer des études originales et hautement intéressantes sur divers sujets. Une des études les plus parlantes de cette section est celle d'Amélie Masson Labonté, qui se penche sur les enjeux entourant les diverses représentations attribuées aux objets patrimoniaux mobiliers par les peuples qui se les approprient. Dans le cas du Sphinx de Bo\azköy opposant l'Allemagne et la Turquie, le conflit de représentation distingue, d'un côté, une Allemagne qui associe les vestiges hittites « à l'exaltation orientaliste autour du mythe aryen » et, de l'autre, une Turquie qui y voit une forme de légitimation pour « la construction de son nouvel État séculier "(22). Ce vestige est alors associé à deux significations nationales distinctes, ce qui rend épineuse la relation entre les deux pays et difficile l'arbitrage nécessaire de l'UNESCO.

Si les parties portant sur le patrimoine immatériel et sur les objets patrimoniaux matériels qui se trouvent au cour du recueil proposent des études dont l'intérêt scientifique est indéniable, la section qui clôture l'ouvrage mérite plus particulièrement que l'on s'y attarde. Les textes y figurant se démarquent puisqu'ils proposent une réflexion analytique et souvent critique des enjeux entourant le patrimoine, en même temps qu'ils se basent sur des cas particuliers pour appuyer leurs propos. Dans un article brillant, Marie-Ėve Breton se penche sur le 
cas de la rue Saint-Malo, à Brest, afin de mettre de l'avant le caractère identitaire du patrimoine. Celui-ci est alors considéré « moins [comme] un lien avec le passé qu'une façon d'envisager l'identité collective au présent et au futur ", bien que ce rapprochement s'accompagne de l'enjeu de "l'hétérogénéité des rapports identitaires » aux biens patrimoniaux (259).

Enfin, cet ouvrage qui regroupe des études s'étendant sur trois continents et qui offre une tribune pour des jeunes chercheurs provenant de divers milieux (même si le tiers est lié à l'UQAM) est une belle lecture pour tous ceux qui réfléchissent aux questions patrimoniales. Même si le matériel est plus représenté que l'immatériel, chacun peut avoir de quoi se satisfaire, notamment avec la quatrième partie qui propose un regard davantage critique. Et malgré les quelques bémols qui parsèment l'ouvrage, il m'apparaît important de souligner la pertinence d'une telle initiative qui contribue à l'élaboration du patrimoine en tant que champ disciplinaire. Comme le soulignent Hébert et Goyette, «[l]e patrimoine est un champ qui se fait en se disant, un objet qui se constitue en s'objectivant, de sorte que les nouveaux entrants dans la discipline sont aussi des pionniers " (2). Son avenir se fait sous nos yeux, et cet ouvrage a le mérite de chercher à participer à ce projet ambitieux.

Louis-Simon Corriveau Université Laval

Ian Russell and Anna Kearney Guigné (eds.). Crossing Over: Fiddle and Dance Studies from Around the North Atlantic 3. (Aberdeen: Elphinstone Institute and St. John's: Memorial University, 2010. Pp. 317, ISBN 9780954568269.)

Kenneth E. Nilsen (ed.). Rannsachadh na Gaidhlig 5: Fifth Scottish Gaelic Research Conference. (Sydney: Cape Breton University Press, 2010. Pp. 366, ISBN 9781897009468.) 